\title{
QUALIDADE DE VIDA EM PESSOAS COM LEISHMANIOSE CUTÂNEA
}

\author{
Quality of life in people with cutaneous leishmaniasis \\ Calidad de vida de personas con leishmaniasis cutánea
}

Artigo Original

\section{RESUMO}

Objetivo: Avaliar a qualidade de vida (QV) de pessoas com leishmaniose cutânea. Métodos: Estudo observacional transversal e descritivo realizado em Brasília, DF, Brasil em 2013 com 44 pacientes portadores de leishmaniose cutânea, por meio da aplicação de um questionário sociodemográfico e clínico e do WHOQOL-bref. Os dados sofreram análises descritivas de frequência, tendência central e dispersão e análise inferencial de comparação entre domínios. Resultados: Os participantes eram em sua maioria do sexo feminino ( $\mathrm{n}=24 ; 54,5 \%$ ), com idade média de 51,80 , casados $(n=23 ; 52,3 \%)$, com primeiro grau incompleto $(n=22 ; 50 \%)$, do $\operatorname{lar}(\mathrm{n}=11 ; 25 \%)$ e procedentes do Distrito Federal $(\mathrm{n}=30 ; 68,2 \%)$. Um total de $27(61,4 \%)$ não possuíam queixas, embora $10(22,7 \%)$ queixaram-se das feridas e $6(13,6 \%)$ de dor. A QV de $36(81,82 \%)$ foi positiva, $30(68,18 \%)$ satisfeitos com a própria saúde, melhores escores observados no domínio relações sociais cujas facetas relações pessoais, apoio social e vida sexual obtiveram mediana 4. Os escores mais baixos foram obtidos no domínio meio ambiente, nas facetas transporte, recursos financeiros e atividade de lazer, com mediana 3. Conclusão: A satisfação no domínio relações sociais representou uma estratégia para o enfrentamento da doença, contribuindo positivamente para a QV dos entrevistados.

Descritores: Qualidade de Vida; Leishmaniose Cutânea; Adulto.

\section{ABSTRACT}

Objective: To assess the quality of life ( $Q o L)$ of people with cutaneous leishmaniasis. Methods: Descriptive cross-sectional study conducted in Brasilia, Distrito Federal, Brazil in 2013 with 44 patients with cutaneous leishmaniasis using sociodemographic and clinical questionnaires and the WHOQOL-bref. Data underwent descriptive analyses of frequency, central tendency and dispersion, and inferential analysis of comparison between domains. Results: Participants were mostly women $(n=24 ; 54.5 \%)$, with a mean age of 51.8 years, married $(n=23 ; 52.3 \%)$, had incomplete primary education $(n=22 ; 50 \%)$, homemakers $(n=11 ; 25 \%)$, and were from Distrito Federal $(n=30 ; 68.2 \%)$. A total of $27(61.4 \%)$ participants did not have any complaints; however, 10 (22.7\%) complained of wounds and $6(13.6 \%)$ complained of pain. The QoL of $36(81.82 \%)$ participants was rated as positive, $30(68.18 \%)$ were satisfied with their own health, better scores were found in the social relationships domain, in which personal relationships, social support and sexual activity facets had a median of 4 . The lowest scores were obtained in the environmental domain, particularly in transport, financial resources and leisure activity facets, with a median of 3. Conclusion: Satisfaction in the social relationships domain represented a strategy for coping with the disease and had a positive effect on the QoL of interviewees.

Descriptors: Quality of Life; Cutaneous Leishmaniasis; Adult.

Recebido em: 19/05/2016 Revisado em: 23/06/2016 Aceito em: 30/09/2016 


\section{RESUMEN}

Objetivo: Valorar la calidad de vida (CV) de personas con leishmaniasis cutánea. Métodos: Estudio observacional, transversal y descriptivo realizado en Brasilia, DF, Brasil en 2013 con 44 pacientes portadores de leishmaniasis cutánea a través de la aplicación de un cuestionario socio demográfico y clínico $y$ del WHOQOL-bref. Fueron realizados análisis descriptivos de frecuencia, tendencia central y dispersión y análisis inferencial para comparación de los dominios. Resultados: Los participantes eran en su mayoría del sexo femenino $(n=24 ; 54,5 \%)$ con media de edad de 51,80 años, casados $(n=23 ; 52,3 \%)$, con educación primaria incompleta $(n=22 ; 50 \%)$, ama de casa $(n=11 ; 25 \%)$ y procedentes del Distrito Federal ( $n=30 ; 68,2 \%)$. Veinte y siete $(61,4 \%)$ no presentaban quejas aunque 10 (22,7\%) se quejaban de las heridas y $6(13,6 \%)$ del dolor. La CV de 36 participantes (81,82\%) fue positiva, 30 (68,18\%) estaban satisfechos con su propia salud y las mejores puntuaciones fueron observadas en el dominio de las relaciones sociales cuyos aspectos de relaciones personales, de apoyo social y de vida sexual presentaron la mediana 4. Las puntuaciones más bajas fueron para los dominios de medio ambiente en los aspectos de transporte, recursos financieros y actividades de ocio con mediana 3. Conclusión: La satisfacción en el dominio relaciones sociales representó una estrategia de afrontamiento de la enfermedad contribuyendo positivamente para la $\mathrm{CV}$ de los entrevistados.

Descriptores: Calidad de Vida; Leishmaniasis Cutánea; Adulto.

\section{INTRODUÇÃO}

As leishmanioses são doenças tropicais de complexa epidemiologia e ecologia, consistindo em um importante problema de saúde pública. São estimados aproximadamente 1 a 1,2 milhões de casos por ano no mundo de sua forma cutânea. Apesar da elevada morbidade, os dados de mortalidade são escassos e geralmente relacionados às internações hospitalares ${ }^{(1)}$.

A incidência é particularmente importante na América Latina, sendo o Brasil o país com maior número de casos novos, ocorrendo em todas as regiões brasileiras, com destaque para as regiões Norte, Nordeste e Centro-Oeste ${ }^{(2)}$. Pela falta de um saneamento ambiental eficiente, com desmatamentos em alta, a doença tem se alastrado pelo Brasil $^{(3)}$. No Distrito Federal, sua ocorrência relaciona-se à inserção de residências próximas às matas e ou a exposição de pessoas em áreas preservadas de mata para atividades de lazer ou ocupacionais ${ }^{(4)}$.

A Leishmaniose Cutânea (LC) caracteriza-se por lesões de pele de diversos espectros clínicos, de apresentação única ou múltipla, que dependerá não só de fatores genéticos e resposta imunitária, como também da espécie de Leishmania envolvida(5). As lesões cutaneomucosas, mesmo após evoluir para a cura, tendem a deixar cicatrizes atróficas, deprimidas, com superfície lisa, áreas de hipo ou hiperpigmentação e traves fibrosas, podendo, algumas vezes, tornarem-se hipertróficas ${ }^{(5)}$. As leishmanioses, portanto, podem assumir grande capacidade destrutiva, desfigurante e incapacitante ${ }^{(5)}$.

Apesar de não ameaçar diretamente à vida, as lesões da leishmaniose podem assumir potencial deformante, afetando as funções sociais e psicológicas dos indivíduos acometidos. Esses podem apresentar crises de ansiedade, depressão, insatisfação com a imagem corporal e diminuição da qualidade de vida. A doença e seu tratamento podem gerar reflexos nas dimensões físicas, psicológicas, sociais e econômicas para quem os experimenta ${ }^{(6)}$.

Qualidade de vida (QV) é um conceito que abrange diversos significados, abordagens teóricas e métodos de medida $^{(7)}$. Na concepção de saúde, o conceito adquire valorização na tentativa de superação do modelo puramente biomédico. As concepções de QV buscam superar o enfoque centrado nos sintomas, na redução da mortalidade ou no aumento da expectativa de vida. Relaciona a percepção do indivíduo em diversos aspectos da vida: físico, ocupacional, psicológico, social e econômico ${ }^{(8-10)}$.

$\mathrm{Na}$ tentativa de criar um conceito com enfoque transcultural, a Organização Mundial de Saúde (OMS) criou o Grupo de Qualidade de Vida (The WHOQOL Group), que definiu QV como “a percepção do indivíduo de sua posição na vida, no contexto da cultura e do sistema de valores em que vive e em relação aos seus objetivos, expectativas, padrões e preocupações." Essa definição relaciona o meio ambiente com aspectos físicos, psicológicos, nível de independência, relações sociais e crenças pessoais ${ }^{(8,9)}$. Em suma, é uma construção subjetiva, multidimensional e composta por elementos de avaliação positivos e negativos. Essa concepção não é imutável, mas está em construção ao longo da vida, sendo passível de modificações globais ou apenas em alguns aspectos ${ }^{(8,11)}$.

Dada à relevância epidemiológica e o impacto dessa doença, faz-se necessário ações de vigilância com o objetivo de reduzir os casos, por meio da identificação e da monitorização das unidades territoriais relevantes, bem como identificação e notificação dos casos. Ademais, ações preventivas e de controle precisam ser estimuladas em ambientes endêmicos ${ }^{(5)}$.

Baseado no exposto, o presente estudo objetiva avaliar a qualidade de vida de pessoas com leishmaniose cutânea.

\section{MÉTODOS}

Trata-se de um estudo observacional de corte transversal e cunho descritivo, desenvolvido com pessoas 
com Leishmaniose Cutânea (LC), em tratamento e acompanhamento no Laboratório de Dermatomicologia do Hospital Universitário de Brasília (HUB), no período de dezembro de 2012 a março de 2013. O HUB é o centro de referência para diagnóstico e tratamento da leishmaniose tegumentar no Distrito Federal, Brasil ${ }^{(5)}$.

Realizaram-se entrevistas, em sala reservada do laboratório, para aplicação de dois instrumentos. O primeiro com objetivo de conhecer aspectos sociodemográficos e clínicos da amostra estudada, baseado em um questionário utilizado em pesquisa com objetivo semelhante ${ }^{(11)}$, e o segundo, o Word Health Organization Quality of Life Instrument Bref (WHOQOL-bref), validado no Brasil ${ }^{(12)}$, para avaliar a qualidade vida de populações adultas.

A amostra, obtida por conveniência, foi composta por 44 pessoas com LC, acessadas durante os quatro meses de coleta de dados e esclarecidas sobre o objetivo e a metodologia do estudo, a garantia do sigilo e anonimato das respostas, bem como, eventuais dúvidas. Entrevistaramse homens e mulheres, maiores de 18 anos, que aceitaram participar do estudo após compreensão das orientações sobre a pesquisa e assinatura do Termo de Consentimento Livre e Esclarecido, conforme estabelece a resolução CNS/ MS n ${ }^{\circ} .466$, de 12 de dezembro de 2012 ${ }^{(13)}$.

O WHOQOL-bref ${ }^{(14)}$ é uma versão abreviada do WHOQOL-100, que surgiu da necessidade de um instrumento mais curto, com menor tempo de preenchimento e que contivesse características psicométricas satisfatórias. Consta de 26 perguntas, duas questões gerais sobre QV e saúde e as demais representam cada uma das 24 facetas que compõem o instrumento original, agrupadas em quatro domínios: físico, psicológico, relações sociais e meio ambiente. As questões levam em conta os valores, aspirações, prazeres e preocupações do entrevistado nos últimos 15 dias. As respostas são contempladas em uma escala do tipo Likert, na qual as perguntas são respondidas por meio de quatro tipos de sequência: intensidade (nadaextremamente), capacidade (nada-completamente), frequência (nunca-sempre) e avaliação (muito insatisfeitomuito satisfeito e muito ruim-muito bom) ${ }^{(14)}$.

Para análise de dados, utilizou-se o programa estatístico SPSS versão 17, incluindo-se análises descritivas de frequência simples, tendência central e dispersão e análise inferencial de comparação entre domínios.

Dos valores encontrados para cada uma das vinte e quatro facetas que compõem os domínios foram obtidas as medianas das respostas, ou seja, o valor que separa $50 \%$ das respostas, quando estas estão ordenadas. Os valores apontam 1 como a pior resposta e 5 a melhor resposta, o que possibilitou verificar quais facetas receberam avaliação positiva ou negativa. Para efeito de uniformização e possibilitando a comparação, analisaram-se de forma invertida os valores apresentados nas facetas relacionadas à dor e ao desconforto, à dependência de tratamentos ou de medicamentos e aos sentimentos negativos, conforme orientação da $\mathrm{OMS}^{(14)}$.

Os escores de avaliação da QV foram calculados separadamente para cada um dos quatro domínios, visto que conceitualmente não está previsto no instrumento um escore global de QV. A pontuação bruta foi transformada para uma escala de 0 a 100 (escore transformado ET 0-100) de acordo com syntax para SPSS proposta pela OMS. Assim, o valor mínimo dos escores de cada domínio é 0 e o máximo é 100, de forma que quanto maior o escore, mais positiva é a avaliação do domínio ${ }^{(14)}$. Com a finalidade de comparar os domínios e verificar diferenças estatisticamente significativas foi realizado o Teste $\mathrm{T}$ de Comparação de Médias para Dados Pareados.

O projeto de pesquisa foi aprovado pelo Comitê de Ética em Pesquisa da Faculdade de Ciências da Saúde da Universidade de Brasília, sob o nº 149/2012, na $10^{\mathrm{a}}$ Reunião Ordinária, realizada no dia 13 de novembro de 2012.

\section{RESULTADOS}

Quanto aos dados sociodemográficos, o estudo contou com a participação de 44 pessoas, 20 do sexo masculino $(45,5 \%)$ e 24 do feminino $(54,5 \%)$. As idades variaram de 30 a 80 anos, com idade média de $51,80 \pm 11,62$. Quanto à escolaridade, $22(50 \%)$ entrevistados possuíam o primeiro grau incompleto ou eram analfabetos e $7(15,91 \%)$ o segundo grau completo. A amostra foi composta por 23 casados (52,3\%), seguidos por 11 solteiros (25\%). Divorciados, vivendo como casados, viúvo e separados somaram 10 participantes (22,7\%). Dentre as ocupações, 11 $(25 \%)$ eram trabalhadores do lar e $10(22,7 \%)$ trabalhadores agropecuários, florestais e da caça e pesca, sendo essas as profissões mais relevantes. Dentre os entrevistados, 30 $(68,2 \%)$ eram procedentes do Distrito Federal (DF).

Quanto aos dados clínicos 27 (61,4\%) entrevistados não possuíam queixas. Quando as possuíam, 10 (22,7\%) queixavam-se das feridas, $6(13,6 \%)$ de dor e apenas 1 $(2,3 \%)$ relatou cansaço extremo.

Nas duas questões iniciais do WHOQOL-bref, relacionadas à percepção geral sobre QV e saúde, 36 $(81,82 \%)$ participantes avaliaram como boa ou muito boa sua QV. Quanto à saúde, $10(22,73 \%)$ estavam nem satisfeitos nem insatisfeitos e $30(68,18 \%)$ afirmaram satisfação ou muita satisfação neste quesito.

Os escores de avaliação dos quatro domínios e das duas questões gerais (avaliação geral) do WHOQOL-bref estão resumidos na Figura 1. 


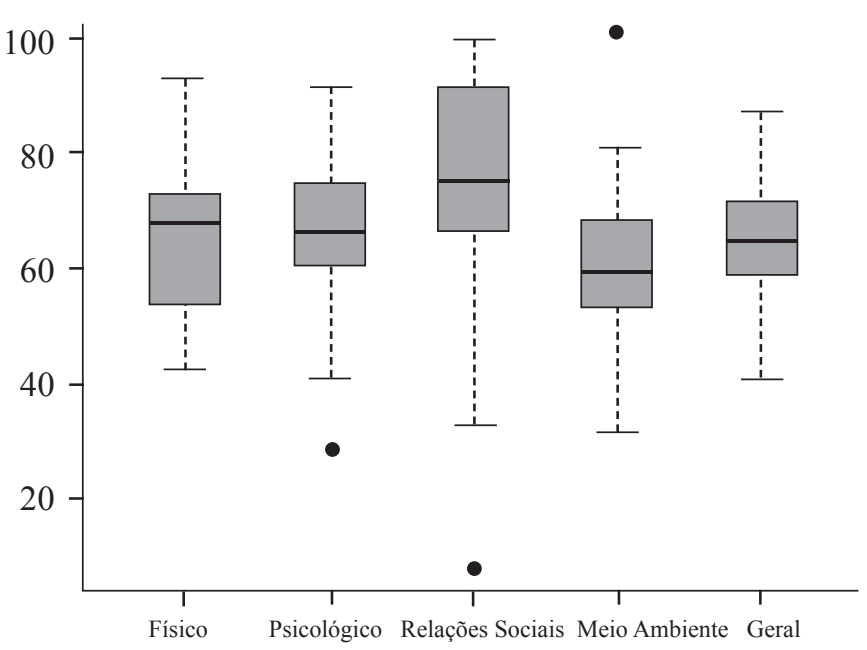

Figura 1 - Escores de avaliação dos domínios da avaliação sobre qualidade de vida em pacientes com leishmaniose cutânea que compõem o WHOQOL-BREF. Brasília, DF, 2013.

Os dados brutos dos domínios apresentaram as seguintes médias e desvios padrão: relações sociais $3,98 \pm 1,09$, psicológico 3,65 $\pm 1,13$, físico 3,59 $\pm 1,12$ e meio ambiente 3,39 $\pm 1,22$.

Já os escores transformados 0-100 (ET 0-100) revelaram as subsequentes médias para os quatro domínios: relações sociais 74,62, psicológico 70,55, físico 61,85 e meio ambiente 59,80 .

Com a finalidade de comparar os domínios e verificar se as diferenças entre eles eram estatisticamente significativas realizou-se o Teste t de Comparação de Médias para Dados

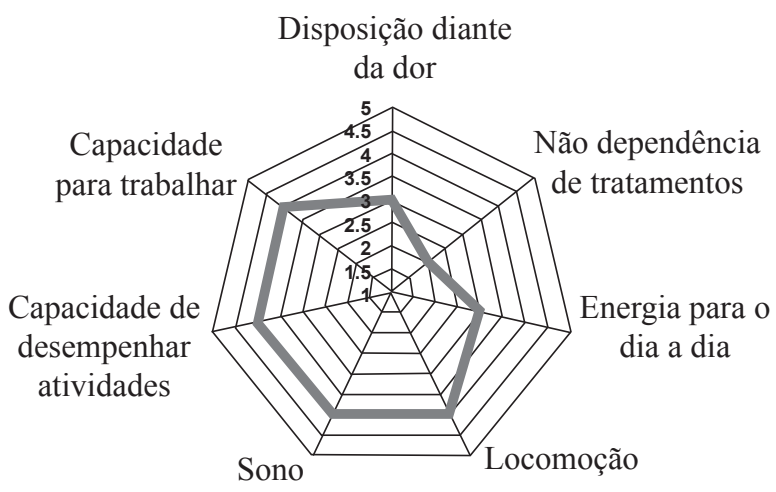

Figura 2 - Mediana das avaliações dos entrevistados nas facetas do domínio físico do WHOQOL-bref. Brasília, DF, 2013.

Do valor encontrado para cada faceta que compõe os domínios foi obtida a mediana das respostas. Os valores apontam 1 como a pior resposta e 5 a melhor resposta. Para efeito de uniformização e possibilitando a comparação, analisaram-se de forma invertida os valores apresentados nas facetas relacionadas à dor e ao desconforto, à dependência de tratamentos ou de medicamentos e aos sentimentos negativos, conforme orientação da $\mathrm{OMS}^{(14)}$.
Pareados. O teste constatou que há diferença entre as dimensões. No entanto, com nível de significância de 5\% não se pode afirmar a diferença entre os domínios relações sociais e psicológicos e nem entre físico e meio ambiente. A dimensão relações sociais foi a melhor avaliada, enquanto o domínio meio ambiente recebeu a pior avaliação.

$\mathrm{Na}$ análise referente à mediana das respostas em cada uma das questões (facetas) de cada domínio, observouse que o domínio físico, representado pela Figura 2, é composto por sete questões que se referem à capacidade de trabalho, capacidade para realizar atividades da vida cotidiana, sono e repouso, mobilidade, energia e fadiga, dor e desconforto e dependência de tratamento médico. A mediana 4 ocorreu em quatro questões (capacidade para trabalhar, capacidade de desempenhar atividades, sono e locomoção) evidenciando que pelo menos metade dos entrevistados estavam satisfeitos nesses quesitos. As questões energia para o dia a dia e disposição diante da dor obtiveram mediana 3, ou seja, metade das avaliações não são boas nem ruins. Dependência de tratamentos obteve mediana 2, nesse item $25(56,82 \%)$ entrevistados referiram necessitar nada ou muito pouco de tratamentos médicos para levar sua vida diária.

No domínio psicológico, composto por seis facetas, todas obtiveram avaliação satisfatória, alcançando mediana 4. Na questão referente à frequência de sentimentos negativos (mau humor, ansiedade, desespero, depressão), $40(90,9 \%)$ entrevistados apresentaram esses sentimentos. Destes, $8(18,18 \%)$ afirmaram experimentar tais sentimentos sempre, $19(43,18 \%)$ muito frequentemente, $9(20,45 \%)$ frequentemente e $4(9,09 \%)$ algumas vezes (Figura 3$)$. 


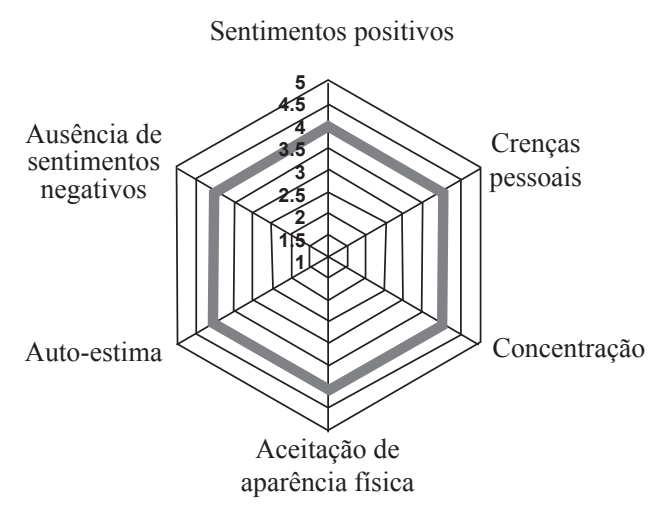

Figura 3 - Mediana das avaliações dos entrevistados nas facetas do domínio psicológico do WHOQOL-bref. Brasília, DF, 2013.

Do valor encontrado para cada faceta que compõe os domínios foi obtida a mediana das respostas. Os valores apontam 1 como a pior resposta e 5 a melhor resposta. Para efeito de uniformização e possibilitando a comparação, analisaram-se de forma invertida os valores apresentados nas facetas relacionadas à dor e ao desconforto, à dependência de tratamentos ou de medicamentos e aos sentimentos negativos, conforme orientação da $\mathrm{OMS}^{(14)}$.

A Figura 4 representa o domínio relações sociais que obteve mediana 4 nos três quesitos avaliados: relações pessoais, apoio social e vida sexual. Tal resultado indica que $22(50 \%)$ participantes estavam satisfeitos em relação ao apoio recebido de familiares e amigos e com suas vidas íntimas.

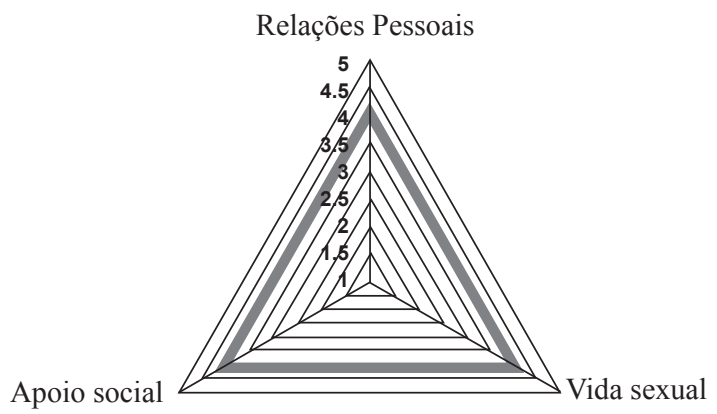

Figura 4 - Mediana das avaliações dos entrevistados nas facetas do domínio relações sociais do WHOQOL-bref. Brasília, DF, 2013.

Do valor encontrado para cada faceta que compõe os domínios foi obtida a mediana das respostas. Os valores apontam 1 como a pior resposta e 5 a melhor resposta. Para efeito de uniformização e possibilitando a comparação, analisaram-se de forma invertida os valores apresentados nas facetas relacionadas à dor e ao desconforto, à dependência de tratamentos ou de medicamentos e aos sentimentos negativos, conforme orientação da $\mathrm{OMS}^{(14)}$.
O domínio meio ambiente, representado pela Figura 5 , possui oito facetas de avaliação e apresentou as piores medidas. Cinco quesitos, segurança física, ambiente físico, acesso aos serviços de saúde, condições de moradia e oportunidade de novas informações, obtiveram mediana 4, relevando que $22(50 \%)$ entrevistados estavam satisfeitos nessas áreas. As facetas meio de transporte, recursos financeiros e atividade de lazer alcançaram mediana 3, ou seja, metade dos participantes mostrou-se nem satisfeita nem insatisfeita em relação a estes itens. Dos entrevistados, apenas $6(13,64 \%)$ afirmaram ter recursos financeiros suficientes para satisfazer completamente as suas necessidades e somente $5(11,36 \%)$ se sentiam completamente satisfeitos ou muito satisfeitos com suas oportunidades de lazer e seus meios de transporte.

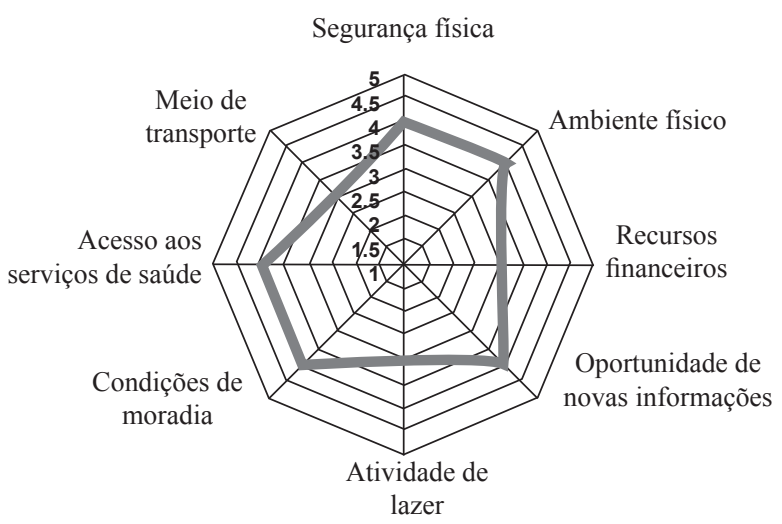

Figura 5 - Mediana das avaliações dos entrevistados nas facetas do domínio meio ambiente do WHOQOL-bref. Brasília, DF, 2013.

Do valor encontrado para cada faceta que compõe os domínios foi obtida a mediana das respostas. Os valores apontam 1 como a pior resposta e 5 a melhor resposta. Para efeito de uniformização e possibilitando a comparação, analisaram-se de forma invertida os valores apresentados nas facetas relacionadas à dor e ao desconforto, à dependência de tratamentos ou de medicamentos $\mathrm{e}$ aos sentimentos negativos, conforme orientação da $\mathrm{OMS}^{(14)}$.

\section{DISCUSSÃO}

A amostra investigada no presente estudo, de $54,5 \%$ de mulheres, não corrobora o perfil brasileiro da doença, que é de predominância masculina $(74 \%)^{(5)}$. Outros estudos sobre qualidade de vida nessa população, no Brasil $^{(15)}$ e na Turquia ${ }^{(16)}$, também evidenciaram predominância do sexo masculino. Já estudos realizados no Irã ${ }^{(16)}$ e no $\operatorname{Marrocos}^{(17)}$ mostraram maior número de mulheres nas amostras pesquisadas. 
As principais ocupações encontradas no atual estudo, do lar $(25 \%)$ e trabalhadores agropecuários, florestais e da caça e pesca $(22,7 \%)$, estão relacionadas às mudanças do perfil de transmissão da doença, representando dois dos perfis epidemiológicos: ocupacional ou lazer e rural ou periurbano. O primeiro está ligado à derrubada desordenada das florestas e às atividades agropecuárias e de turismo, enquanto que o segundo se relaciona com áreas de colonização próximas às matas residuais ${ }^{(5)}$.

Estudos no Irã ${ }^{(6)}$ e na Turquia ${ }^{(16)}$ não encontraram relação entre sexo, estado civil, ocupação, nível educacional e QV. A aparência e o tipo de lesão, no entanto, demonstraram influência sobre a vida e QV, sendo as lesões ulcerativas as que mais significativamente a modificaram ${ }^{(6)}$.

No presente estudo, nos aspectos clínicos, as principais queixas também estiveram relacionadas às lesões e à dor. A influência psicossocial das doenças de pele está diretamente ligada a quão visíveis elas são. As lesões da LC ocorrem particularmente em áreas expostas do corpo, podendo ser, muitas vezes, deformantes. Essas particularidades da localização e da gravidade da lesão podem levar o indivíduo a se sentir estigmatizado, afetando não só sua autoestima, como seu bem-estar social ${ }^{(16)}$.

Sentimentos negativos (mau humor, ansiedade, desespero, depressão), do domínio psicológico, estiveram presentes em $90,9 \%$ dos entrevistados do presente estudo. As lesões de pele frenquentemente estão associadas à depressão, à ansiedade, à baixa autoestima e à satisfação pessoal, bem como, à ideação suicida ${ }^{(16)}$. Estudo pioneiro sobre a influência da LC na QV mostrou aumento dos sintomas de ansiedade e de depressão nessa população ${ }^{(18)}$. Pesquisa posterior demonstrou maiores níveis de depressão em crianças e em adolescentes com $\mathrm{LC}^{(16)}$. Como não ameaçam diretamente a vida, as consequências psicológicas das doenças dermatológicas são muitas vezes negligenciadas ${ }^{(16)}$. No entanto, o reconhecimento precoce do início dos sintomas e o desenvolvimento de ações de saúde a fim de minimizá-los são importantes para melhorar a qualidade de vida. Além de assegurar uma melhor resposta ao tratamento ${ }^{(16)}$.

Os resultados apresentados no domínio relações sociais demonstram que os entrevistados do presente estudo recebem apoio familiar e social e apresentam uma vida sexual satisfatória. As relações sociais de pacientes com LC em outros estudos também foram influência positiva na qualidade de vida ${ }^{(6,15)}$.

$\mathrm{O}$ alto número de participantes insatisfeitos, na atual pesquisa, com recursos financeiros, acesso às atividades de lazer e meios de transporte traduz suas dificuldades socioeconômicas. As doenças negligenciadas prevalecem em pessoas com baixo nível socioeconômico e agravam as desigualdades no Brasil ${ }^{(5,19)}$.
Ações de vigilância e controle são necessárias para redução dos casos de $\mathrm{LC}$ e do seu impacto na qualidade de vida dos indivíduos afetados ${ }^{(5)}$. A prevenção da $\mathrm{LC}$ tem sido um problema de difícil controle nas regiões endêmicas, uma vez que correlaciona fatores ecológicos-ambientais, socioeconômicos, culturais e comportamentais ${ }^{(20)}$.

O desenvolvimento de políticas de saúde que proporcionem melhorias na prevenção e diagnóstico e tratamento adequados é fundamental. Essas ações devem envolver ativamente a comunidade ${ }^{(5,21)}$. Como cada região tem características epidemiológicas particulares, é fundamental que as ações sejam baseadas nessas peculiaridades $^{(21)}$.

A falta de informações sobre a transmissão e o tratamento dificultam as estratégias de prevenção. $\mathrm{O}$ conhecimento sobre a enfermidade é, muitas vezes, restrito às pessoas que já tiveram a doença ou àquelas que tiveram casos na família ou próximos ${ }^{(21)}$. O diagnóstico precoce e o tratamento adequado dos casos humanos devem ser priorizados ${ }^{(5,20)}$. Portanto, a educação em saúde deverá ser voltada para a epidemiologia, a transmissão, o reconhecimento dos sinais clínicos e o tratamento ${ }^{(5,21)}$. Para tanto, é preciso que haja articulação no campo político e acadêmico, no âmbito da gestão, no processo de atenção e de trabalho em saúde e no controle social ${ }^{(20)}$.

Há mais de 50 décadas o tratamento da LC é baseado nos antimoniais pentavalentes. Apesar da eficácia, esses medicamentos apresentam efeitos colaterais importantes e custos elevados. Além de exigirem uso endovenoso, o tempo de cura das lesões é prolongado, o que interfere na vida e na qualidade de vida das pessoas que desenvolvem a doença. A literatura recomenda a necessidade de estudos que avaliem novos regimes terapêuticos, com o objetivo de encontrar um medicamento tão eficaz quanto os antimoniais, porém de fácil administração e baixa toxicidade. Como a LC é uma doença de países em desenvolvimento não há, no entanto, incentivos comerciais para as indústrias farmacêuticas desenvolverem drogas baratas e eficazes para o tratamento ${ }^{(22)}$.

As ações de vigilância da leishmaniose incluem não só o reconhecimento e a notificação dos casos, como também a identificação de áreas de transmissão, o reconhecimento das espécies de flebotomíneos e controle desses $^{(5)}$. Estudo realizado no Marrocos ${ }^{(17)}$ demonstrou a diminuição da incidência da doença após integração dos órgãos responsáveis, em nível nacional, pela saúde e pela agricultura no delineamento de estratégias de controle dos vetores nas áreas afetadas.

Medidas preventivas devem ser estimuladas. Elas estão relacionadas à proteção individual em áreas de transmissão e ao manejo ambiental para controle dos vetores. Dentre as medidas de proteção individual em áreas endêmicas, 
destacam-se uso de repelentes, evitar exposição nos horários de atividade do vetor, uso de mosquiteiro de malha fina, bem como telagem de portas e janelas ${ }^{(5)}$.

São necessários estudos mais detalhados nessa área. Uma limitação da presente pesquisa foi a utilização de um instrumento de avaliação de qualidade de vida genérico. Se por um lado esse instrumento capta questões gerais (físicas, psicológicas, sociais e do meio ambiente) não aprofunda no tema saúde/doença, no caso leishmaniose cutânea, na avaliação da qualidade de vida. Outros estudos poderiam construir e validar instrumento específico para avaliação da qualidade de vida em pessoas com a doença. Uma vertente a ser explorada é a comparação durante e após o tratamento/acompanhamento da patologia para verificar se ocorreram mudanças na percepção da qualidade de vida. Paralelamente, é possível utilizar metodologias qualitativas, pois qualidade de vida é um tema subjetivo e as metodologias poderiam ser complementares. Espera-se que esse conhecimento permita a valorização das percepções dessa população em relação às diversas áreas de sua vida e, desse modo, aspectos prioritários para a prevenção, o tratamento e o acompanhamento da doença possam ser identificados e alcançados.

\section{CONCLUSÃO}

O presente estudo permitiu conhecer a avaliação da qualidade de vida de pessoas com leishmaniose cutânea em tratamento e acompanhamento no Hospital Universitário de Brasília. A satisfação no domínio relações sociais representou uma estratégia para o enfrentamento da doença, contribuindo positivamente para a QV dos entrevistados. O domínio meio ambiente teve influência negativa na avaliação dos participantes, principalmente devido às facetas de recursos financeiros, atividade de lazer e transporte, evidenciando a condição vulnerável dessa população acometida por uma doença negligenciada.

\section{REFERÊNCIAS}

1. Alvar J, Vélez ID, Bern C, Herrero M, Desjeux P, Cano $\mathrm{J}$ et al. Leishmaniasis worlwide and global estimates of its incidence. PloS One. 2012;7(5):e35671.

2. Batista FMA, Machado FFOA, Silva JMO, Mittmann J, Barja PR, Simioni AR. Leishmaniose: perfil epidemiológico dos casos notificados no estado do Piauí entre 2007 e 2011. Rev UNIVAP 2014;20(35): 44-55.

3. Oliveira JVS, Magalhães SCM, Cardoso AF, Lopes JO, Laughton BA. Promoção da saúde: a importância do saneamento ambiental e sua influência na ocorrência da leishmaniose tegumentar em Montes Claros/MG [resumo]. In: XIV Colóquio Ibérico de Geografia; 2014 Nov 11-14; Guimarães [acesso em 2016 Maio 15] Disponível em: http://www.lasics. uminho.pt/conferences/index.php/CEGOT/XIV_ ColoquioIbericoGeografia/paper/view/1994

4. Carvalho MSL, Bredt A, Meneghin ERS, Oliveira C. Flebotomíneos (Diptera: Psychodidae) em áreas de ocorrência de leishmaniose tegumentar americana no Distrito Federal, Brasil, 2006 a 2008. Epidemiol Serv Saúde. 2010;19(3):227-37.

5. Ministério da Saúde (BR). Manual de vigilância da leishmaniose tegumentar americana. $2^{\mathrm{a}}$ ed. atual. Brasília: Ministério da Saúde; 2010.

6. Vares B, Mohseni M, Heshmatkhah A, Farjzadeh S, Safizadeh H, Shamsi-Meymandi S, et al. Quality of life in Patients with Cutaneous Leishmaniasis. Arch Iran Med. 2013;16(8):474-7.

7. Silva PAB, Soares SM, Santos JFG, Silva LB. Ponto de corte para o WHOQOL-BREF como preditor de qualidade de vida em idosos. Rev Saúde Pública. 2014;48(3):390-7.

8. Minayo MCS, Hartz ZMA, Buss PM. Qualidade de vida e saúde: um debate necessário. Ciênc Saúde Coletiva. 2000;5(1):7-18.

9. Power M. Qualidade de vida: visão geral do projeto WHOQOL. In: Fleck MPA. A avaliação de qualidade de vida: guia para profissionais de saúde. Porto Alegre: Artmed; 2008. p. 48-59.

10. Castro MMLD, Hokerberg YHM, Passos SRL. Validade dimensional do instrumento de qualidade de vida WHOQOL-BREF aplicado a trabalhadores da saúde. Cad Saúde Pública. 2013;29(7):1357-69.

11. Bampi LNS, Guilhem D, Lima DD. Qualidade de vida em pessoas com lesão medular traumática: um estudo com o WHOQOL. Rev Bras Epidemiol 2008;11(1): 67-77.

12. Fleck MPA, Louzada S, Xavier M, Chachamovich E, Vieira G, Santos L, et al. Aplicação da versão em português do instrumento abreviado de avaliação de qualidade de vida "WHOQOL-bref ". Rev Saúde Pública. 2000;34(2):178-83.

13. Ministério da Saúde (BR). Resolução $n^{\circ}$ 466, de 12 de dezembro de 2012. Aprova as diretrizes e normas regulamentadoras de pesquisas envolvendo seres humanos [estatuto na internet]. Diário Oficial da República Federativa do Brasil, Brasília (DF), 2012 dez 12; Seção 1:137. 
14. Chachamovich E, Fleck MPA. Desenvolvimento do WHOQOL-bref. In: Fleck MPA. A avaliação de qualidade de vida: guia para profissionais de saúde. Porto Alegre: Arted; 2008. p. 74-82.

15. Toledo AC Jr, Silva RE, Carmo RF, Amaral TA, Luz ZM, Rabello A. Assessment of the quality of life of patients with cutaneous leishmaniasis in Belo Horizonte, Brazil, 2009-2010. A pilot study. Trans R Soc Trop Med Hyg. 2013;107(5):335-6.

16. Turan E, Kandemir H, Yesilova Y, Ekisin S, Tanrikulu $\mathrm{O}$, Kandemir SB, et al. Assessment of psychiatric morbidity and quality of life in children and adolescents with cutaneous leishmaniasis and their parents. Postepy Dermatol Alergol. 2015;32(5):344-8.

17. Bennis I, Brouwere V, Ameur B, Laamrani AEI, Chichaoui S, Hamid S, et al. Control of cutaneous leishmaniasis caused by Leishmania major in southeastern Morocco. Trop Med Int Health. 2015, 20(10):1297-305.

18. Yanik M, Gurel MS, Simsekt Z, Kati M. The psychological impact of cutaneous leishmaniasis. Clin Exp Dermatol. 2004;29(5):464-7.

19. Penna GO, Domingues CMAS, Siqueira JB Junior, Elkhoury ANSM, Cechinel MP, Grossi MAF, et al. Doenças dermatológicas de notificação compulsória no Brasil. An Bras Dermatol. 2011;86(5):865-77.
20. Santos JLC, Melo MB, Ferreira RA, Fonseca AFQ, Vargas MLF, Gontijo CMF. Leishmaniose tegumentar americana entre os indígenas Xakriabá: imagens, ideias, concepções e estratégias de prevenção e controle. Saúde Soc. 2014,23(3):1033-48.

21. Ramos JVA. Plano de intervenção para implantação de ações educativas de prevenção e controle da leishmaniose tegumentar americana no Distrito de Três Ladeiras - Igarassu - PE. [monografia de especialização]. Recife: Centro de Pesquisas Aggeu Magalhães - FioCruz; 2011.

22. Almeida OLS, Santos JB. Avanços no tratamento da leishmaniose tegumentar do novo mundo nos últimos dez anos: uma revisão sistemática da literatura. An Bras Dermatol. 2011;86(3):497-506.

\section{Endereço para correspondência:}

Isabel de Melo Honório

Universidade de Brasília - UNB

Faculdade de Ciências da Saúde

Campus Darcy Ribeiro s/n

Bairro: Asa Norte

CEP: 70910-900 - Brasília - DF - Brasil

E-mail: isabelm.honorio@gmail.com 